\title{
INCORPORATING THE STRENGTH OF MIP MODELING IN SCHEDULE CONSTRUCTION
}

\author{
Cor A.J. Hurkens ${ }^{1}$
}

\begin{abstract}
Linear programming techniques can be used in constructing schedules but their application is not trivial. This in particular holds true if a trade-off has to be made between computation time and solution quality. However, it turns out that - when handled with care - mixed integer linear programs may provide effective tools. This is demonstrated in the successful approach to the benchmark constructed for the 2007 ROADEF computation challenge on scheduling problems furnished by France Telecom.
\end{abstract}

Keywords. Scheduling, integer programming, lower bounds, hybrid methods.

Résumé. L'application de techniques programmation linéaire pour la résolution de problème d'ordonnancement n'est pas trivial, particulièrement lorsque qu'un compromis entre qualité de la solution fournie et temps de calcul est recherché. Dans ce cas des heuristiques peuvent être couplées pour améliorer les performances des modèles de programmation linéaire. La combinaison de telles méthodes a montré son efficacité dans le cadre de la résolution du Challenge ROADEF 2007.

Mots Clés. Planification, programmation en nombres entiers, bornes inférieures, hybridisation.

Mathematics Subject Classification. 90C11, 90B35.

\footnotetext{
Received December 1, 2008. Accepted June 17, 2009.

${ }^{1}$ Eindhoven University of Technology, Department of Mathematics and Computer Science, P.O. Box 513, 5600 MB Eindhoven, The Netherlands; wscor@win.tue.nl 


\section{INTRODUCTION}

In this paper we present an approach based on polyhedral techniques for solving a complex scheduling problem. The working example is the France Telecom technician assignment problem, posed as a challenge for the ROADEF 2007 competition.

Linear programming techniques are found in the literature concerning sequencing and scheduling but not in great abundance. For instance they show up for tackling relatively simple problems such as minimizing maximum lateness for a preemptive schedule of jobs with release times on unrelated machines [2]. Some production application can also be found [3], as well as computational studies [1]. Also linear programming models with a combinatorial structure such as flows or matchings can be fruitful in some scheduling contexts. It is very tempting to use complete integer linear models for scheduling problems in practical applications. However, it turns out that more than often, an integer linear programming model will at best describe formally the underlying problem correctly, while it does not provide any solution when passed onto a solver. We have taken this opportunity to test how the strength of MIP modeling can be applied - in practice - to reallife scheduling challenges. We developed a schedule constructing algorithm with a lot of subroutines that make use of a CPLEX-library for solving LP and ILP sub-problems.

The remainder of this paper discusses an analysis of the scheduling problem that pinpoints the various sections for which a polyhedral approach maybe useful, and proceeds by giving for the identified parts an appropriate MIP model.

\section{Analyzing the France Telecom technician dispatching PROBLEM}

\subsection{BRIEF DESCRIPTION}

The scheduling problems in the 2007 ROADEF challenge were furnished by France Telecom and involved the daily formation of teams of skilled technicians and the assignment of tasks to teams over a certain scheduling period. The input for each problem is a list of tasks to be scheduled, and a list of technicians that in conjunction with other technicians - may carry out those tasks. The output is a schedule which for each day describes a partition of the technicians into teams, an assignment of some tasks to these teams, and for each scheduled task a processing time interval. In the overall schedule, some tasks may not be scheduled at all, but then the total cost of these abandoned tasks may not exceed the predefined budget. If a task is being abandoned then all its successor tasks must be abandoned as well. The schedule has to obey certain restrictions regarding the expertise of the formed teams, and the order in which tasks are being carried out. Tasks have to be carried out without interruption, in the course of one day. A team can handle only one task at a time, and stays together for the duration of a day. A team can 
TABLE 1. Characteristics of France Telecom problems.

\begin{tabular}{|l|rrrrr|r|rrrr|}
\hline Inst & Jobs & Techs & Reqs & Prec & LB & Cost & $C_{\max }^{1}$ & $C_{\max }^{2}$ & $C_{\max }^{3}$ & $C_{\max }^{4}$ \\
\hline A1 & 5 & 5 & 6 & 0 & 2265 & 2340 & 60 & 15 & 90 & - \\
A2 & 5 & 5 & 6 & 2 & 2055 & 4755 & 135 & - & 195 & - \\
A3 & 20 & 7 & 6 & 0 & 11310 & 11880 & 300 & 120 & 360 & - \\
A4 & 20 & 7 & 12 & 7 & 10629 & 13620 & 210 & 360 & 540 & - \\
A5 & 50 & 10 & 6 & 13 & 26910 & 29355 & 855 & 240 & 300 & - \\
A6 & 50 & 10 & 20 & 11 & 17625 & 20280 & 525 & 120 & 780 & - \\
A7 & 100 & 20 & 20 & 31 & 28442 & 32520 & 600 & 780 & 960 & - \\
A8 & 100 & 20 & 20 & 21 & 16191 & 18960 & 480 & 180 & 600 & - \\
A9 & 100 & 20 & 20 & 22 & 25553 & 28320 & 720 & 240 & 960 & - \\
A10 & 100 & 15 & 20 & 31 & 36399 & 40650 & 1020 & 435 & 1200 & - \\
\hline B1 & 200 & 20 & 16 & 47 & 32085 & 35460 & 420 & 1005 & 1755 & 2610 \\
B2 & 300 & 30 & 15 & 143 & 14296 & 18300 & 450 & 165 & 615 & 930 \\
B3 & 400 & 40 & 16 & 57 & 14610 & 16965 & 195 & 480 & 870 & 1305 \\
B4 & 400 & 30 & 120 & 112 & 16635 & 27015 & 645 & 240 & 1005 & 1575 \\
B5 & 500 & 50 & 28 & 427 & 45060 & 94200 & 1620 & 2310 & 3060 & 4260 \\
B6 & 500 & 30 & 24 & 457 & 24180 & 30510 & 750 & 285 & 1035 & 1380 \\
B7 & 500 & 100 & 50 & 387 & 25290 & 33060 & 720 & 480 & 1080 & 1860 \\
B8 & 800 & 150 & 40 & 440 & 31920 & 32160 & 480 & 840 & 1230 & 2040 \\
B9 & 120 & 60 & 25 & 55 & 25681 & 28080 & 720 & 360 & 480 & 960 \\
B10 & 120 & 40 & 25 & 55 & 32790 & 35040 & 960 & 360 & 480 & 1200 \\
\hline X1 & 600 & 60 & 60 & 195 & 87990 & 151980 & 2160 & 4500 & 5580 & 6180 \\
X2 & 800 & 100 & 36 & 536 & 6075 & 9090 & 210 & 105 & 330 & 420 \\
X3 & 300 & 50 & 60 & 224 & 37950 & 50400 & 720 & 1440 & 1920 & 2400 \\
X4 & 800 & 70 & 105 & 321 & 61130 & 65640 & 1650 & 480 & 2460 & 2880 \\
X5 & 600 & 60 & 60 & 201 & 86145 & 147000 & 1980 & 5340 & 2760 & 5760 \\
X6 & 200 & 20 & 36 & 128 & 6180 & 10440 & 240 & 90 & 480 & 540 \\
X7 & 300 & 50 & 60 & 235 & 26220 & 33120 & 510 & 720 & 1680 & 2040 \\
X8 & 100 & 30 & 105 & 40 & 19050 & 23580 & 540 & 240 & 990 & 1140 \\
X9 & 500 & 50 & 60 & 184 & 86520 & 136020 & 1980 & 3960 & 4920 & 5460 \\
X10 & 500 & 40 & 60 & 184 & 97950 & 131700 & 1920 & 3780 & 4920 & 5340 \\
\hline
\end{tabular}

handle a task only then, when the cumulative skills of the technicians in the team dominate the skills required by the task. Tasks can be handled not earlier than at the latest completion time of all its predecessors.

The quality of a schedule is determined by the weighted sum of completion times of the various priority classes. Each task belongs to some priority class, where higher priority classes evidently get a higher weight. A priority class is considered completed once all of its unabandoned tasks have been completed.

The principal characteristics and results for the thirty Challenge problems are given in Table 1. In the course of the challenge, three benchmarks were provided. The first one, $A$, was a set of relatively small problems. In this set no tasks could 
be abandoned. Set $B$ contains real-life problems, or at least problems of reallife size. These two sets were used to fine-tune the algorithms developed for the challenge. Finally, there was a set $X$ of problems that would be used for the final ranking. They were not disclosed until after all algorithms had been submitted and tested. Their complexity was to be comparable to those in set $B$.

In Table 1 we give for each of the test instances the number of jobs, the number of technicians, the number of skill requirements, and the number of immediate precedences between jobs. Moreover we present a lower bound on the objective function, based on a relaxation described in a later section. In the right half of the table we give the values of the best solution found. The value of each schedule is a certain weighted combination of 3 or 4 priority-makespans. Here $C_{\max }^{p}$ denotes the latest completion time of a job in the schedule belonging to priority class $p$. Most of these were obtained by running the generic implementation of our algorithm for a period of $40 \mathrm{~min}$ or less. For the $X$-instances a time limit of $20 \mathrm{~min}$ was used.

It can be seen from the table that the instances are varying a lot. The $A$-instances are relatively small and do not allow to reject (or outsource) jobs. The $B$-instances allow for outsourcing jobs (under restrictions) and some of them have a lot of precedences between jobs. Furthermore, in some $B$-instances the number of requirements may be rather high.

\subsection{Notation AND Key OBSERVATIONS}

In the remainder tasks will be indexed by $j$. A task $j$ has a duration of $d(j)$ time units where a working day consists of 120 time units. A task must be processed without interruption by a team of technicians, within a working day. Task $j$ has a priority $P(j) \in\{1,2,3,4\}$, where a low number denotes a high priority. In case task $j$ has to be finished before task $k$ can be started we denote this by $j \rightarrow k$, and we say that $j$ is a predecessor of $k$ and $k$ is a successor of $j$. In a schedule let $\mathrm{CT}(j)$ denote the completion time of task $j$ in time units. Then, for $p \in\{1,2,3,4\}$, the priority makespan of priority $p, C_{\max }(p)$ or $C_{\max }^{p}$ is defined as the maximum completion time $\mathrm{CT}(j)$ over all unabandoned jobs with $P(j)=$ $p$. Each priority is weighted by a number $W(p)$. In all instances we used the weights $W(1)=28, W(2)=14, W(3)=4$, and $W(4)=0$. Moreover the overall makespan denoted by $C_{\max }^{0}$ or $C_{\max }(0)$ and defined by $C_{\max }^{0}=\max _{p \in\{1,2,3,4\}} C_{\max }^{p}$ was weighted by a factor $W(0)=1$. Hence, the value of a schedule is given by $\sum_{p \in\{0,1,2,3,4\}} W(p) C_{\max }^{p}$.

Technicians are indexed by $t$ and days are indexed by $d \in\{0,1, \ldots\}$. Day $d$ is a time interval starting at time $120 d$ and ending at $120(d+1)$. A technician $t$ may or may not be available on day $d$. By $\mathrm{Amd}_{d}$ we denote the number of technicians available on day $d$. By $\mathrm{Cmd}_{d}$ we denote the cumulative number of man-days up to and not including day $d$. These numbers will be useful when computing bounds on priority makespan values.

Teams will be composed for each day in the schedule, where only technicians that are available can be assigned. On each day a technician is assigned to at most 
one team. Teams will be indexed by $\tau$. A team can carry out a task only if it contains enough competent technicians.

In the France Telecom problems, competence was described in terms of skills, domains and levels. Each instance was characterized by $D$ skill domains, and within each domain, we consider $L$ levels of expertise. For each technician $t$ we are given his competence level $\operatorname{Comp}(t, s) \in\{0,1, \ldots, L\}$ in skill domain $s$. Here a level 0 denotes no competence at all, and a level $L$ refers to a real expert. For the processing of a task $j$ the necessary team competence is described by a number $\operatorname{Req}(j, s, \ell)$ denoting the minimum number of technicians in the team that have a competence level at least $\ell$ in skill domain $s$. Note that this definition implies a cumulative notion, hence we have $\operatorname{Req}(j, s, \ell) \geq \operatorname{Req}(j, s, \ell+1)$. In the following linear programming models it is much easier to account for competence requirements by using a slightly different but equivalent way of bookkeeping competence levels and requirements. We have $D * L$ pairs, and we use an index $i \in\{1, \ldots, D * L\}$ to refer to these pairs. For each pair $i=(s, \ell)$ we denote by a $0 / 1$ value $S_{i}^{t}$ whether or not a technician $t$ contributes to skill domain $s$ at level $\ell$ or not. Here a value $S_{i}^{t}=1$ denotes that $t$ has skill level $\ell$ or higher, in domain $s$. For $i=(s, \ell)$ we use $R_{i}^{j}=\operatorname{Req}(j, s, \ell)$ to denote the required level of competence of a team handling $j$. So team $\tau$ can handle task $j$ if and only if $\sum_{t \in \tau} S_{i}^{t} \geq R_{i}^{j}$, for all $i$. In the remainder one can view $i$ as a skill that a technician has or has not. The numbers of required skills as mentioned in Table 1 are the products $D L$.

As already indicated, it is not always the case that an optimal solution is reached by processing all tasks in order of priority. In the following, both in the constructing heuristics, as well as in the determination of lower bounds on the schedule cost, we have to prefix the order by which priority classes will be completed. As priority class 4 does not have a positive weight, it suffices to distinguish between solutions in which priority classes are completed in order $\pi_{1}, \pi_{2}, \pi_{3}, \pi_{4}, \pi_{5}$ where $\left(\pi_{1}, \pi_{2}, \pi_{3}\right)$ is some permutation of $1,2,3$, and where $\pi_{4}=4$ and $\pi_{5}=0$. For a given ordering we say that task $j$ contributes to the makespan of $\pi_{m}$, if it belongs to priority class $\pi_{1}, \ldots, \pi_{m-1}$ or $\pi_{m}$, or if it has a successor task that contributes to $\pi_{m}$. We should only count its contribution if the task is not abandoned.

\section{Solution STRATEGy}

To have some idea how the solution will look like, and to have an a posteriori solution quality guarantee, we first compute a lower bound on the value of the schedule cost. The lower bound is based on a relaxation of the problem by allowing preemption, and by estimating the minimum number of technicians needed for a job. Next we construct a solution, partially based on the relaxed schedule. Since we have four priority classes, three of which having a non-zero weight, we consider schedules with a fixed order for the makespans $C_{\max }^{1}, C_{\max }^{2}$, and $C_{\max }^{3}$. Note that it may be profitable to process first all jobs of say priority 2 , and then the jobs of priority 1. This is particularly true if there are only few jobs of priority class 2 . The table with solutions indeed shows very good solutions with makespans in 
other orders than $1,2,3,4$. We therefore consider all 6 permutations of priority classes $1,2,3$.

\subsection{Preprocessing}

One of the ingredients in the heuristics developed is the estimation of the size of teams handling some task $j$. For this we use the vector of required skills, and compare it to the skills provided by the technicians. The skill requirement of a task $j$ is expressed by a vector $\left(R_{i}^{j}\right)_{i \in S}$ where $R_{i}^{j} \in \mathbb{Z}_{+}$denotes the required number of technicians in the team having skill $i$. Obviously, the value $\max _{i} R_{i}^{j}$ is a lower bound on the size of a team handling task $j$. However, it may very well be the case that no expert has both skill $x$ and skill $y$. If a task requires a team with one expert for $x$ and one expert for $y$, such a team then consists of at least two experts, even if we have $\max _{i} R_{i}^{j}=1$. To find the exact minimum of technicians needed to handle task $j$ we solve for each task $j$ a Mixed Integer Programming problem. Here, technician $t$ offers skills $\underline{S}^{t} \in\{0,1\}^{n}$, and task $j$ requires skills $\underline{R}^{j} \in\{0,1,2, \ldots\}^{n}$.

Minimum number of technicians in team doing job $j$ :

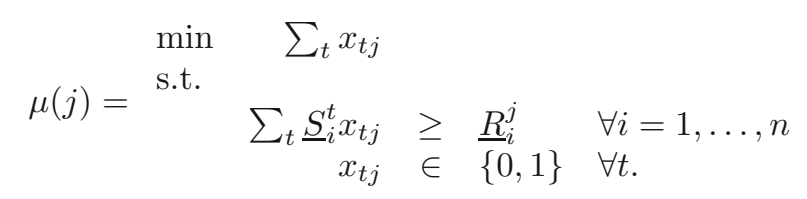

Evidently, $\mu(j) \geq \max _{i} \underline{R}_{i}^{j}$.

\subsection{LOWER BOUND}

Consider a permutation $\pi_{1}, \pi_{2}, \pi_{3}$ of $1,2,3$, and define $\pi_{4}=4, \pi_{5}=0$. For a given order of the makespans, $C_{\max }\left(\pi_{1}\right) \leq C_{\max }\left(\pi_{2}\right) \leq C_{\max }\left(\pi_{3}\right) \leq C_{\max }(4) \leq$ $C_{\max }(0)$, and a given set of jobs to schedule, a lower bound on $C_{\max }\left(\pi_{m}\right)$ is found by calculating the total amount of man-days (technician-units) $M D$ needed to process all jobs that should be finished by time $C_{\max }^{\pi_{m}}$, and computing the first time $T$ at which this number of man-days has been made available. If at each day $\mu$ technicians are available $T=\frac{M D}{\mu}$ days. If the number of available technicians varies per day (which is the case here) a simple adjustment is needed. See figure 1 for the more general case.

To estimate the number of technicians needed for a task $j$ we use the lower bound $\mu(j)$ obtained in Section 2.1. By multiplication with the duration, $d(j)$, and division by 120 , it is converted in total required man-days. Lower bounds on the makespans are computed simultaneously. In case it is considered to abandon jobs it is really fruitful to formulate the lower bound problem as an integer linear programming problem. The following Mixed Integer Program models the general minimum cost of a preemptive schedule, providing enough man-days to carry 


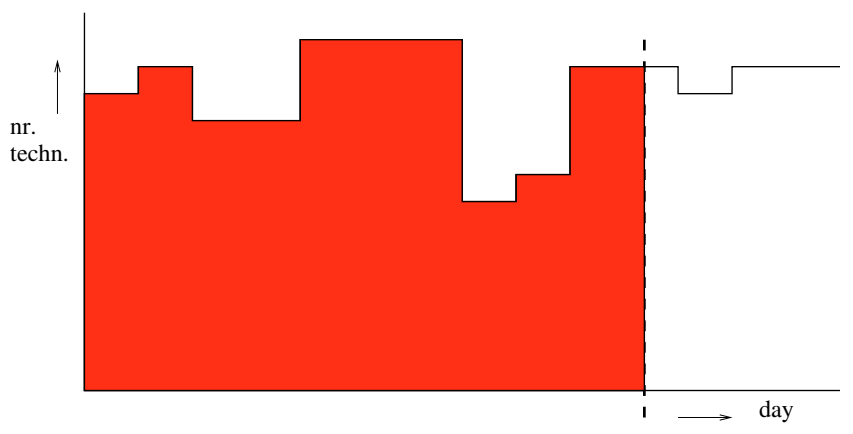

Figure 1. Profile of man-days offered.

out the selected jobs. The variables $C\left(\pi_{m}\right)$ denotes the priority class makespan, expressed in time-units. Considering the order of priority classes, let $a_{m j}$ denote the amount of man-days that task $j$ contributes to makespan $C\left(\pi_{m}\right)$. Let $\operatorname{Rmd}_{m}=\sum_{j} a_{m j}$ denote the total man-days of tasks $j$ contributing to $C\left(\pi_{m}\right)$. The variable $X_{j}$ indicates whether a task $j$ is abandoned $\left(X_{j}=1\right)$ or not $\left(X_{j}=0\right)$. The cost of abandoning task $j$ is given by $\mathrm{Abc}_{j}$, whereas Budget is an upper bound on the cost spent on abandoning tasks. For a proper lower bound on the schedule cost, we must take coefficients $F_{j}=0$. This coefficient is only introduced to allow for variations on preemptive schedules, as it turns out that there can be difficult tasks that one may want to abandon with high preference.

Minimize $\sum_{m} W\left(\pi_{m}\right) C_{\max }\left(\pi_{m}\right)+\sum_{j} F_{j} X_{j}$ subject to:

$$
\begin{array}{rlrl}
C_{\max }\left(\pi_{m}\right) & \leq C_{\max }\left(\pi_{m+1}\right) & & \forall m<5 \\
C_{\max }\left(\pi_{m}\right) & \geq 120 * d * Z_{m d}+120 Y_{m d} & \forall m, d \\
\sum_{d} Z_{m d} & =1 & & \forall m \\
Y_{m d} & \leq Z_{m d} & & \forall m, d \\
\sum_{d}\left(\mathrm{Cmd}_{d} Z_{m d}+\operatorname{Amd}_{d} Y_{m d}\right) & \geq \mathrm{Rmd}_{m}-\sum_{j} a_{m j} X_{j} & & \forall m \\
Z_{m d} & \in\{0,1\} & \\
Y_{m d} & \geq 0 & \\
\sum \mathrm{Abc}_{j} X_{j} & \leq \text { Budget } & & \forall j \rightarrow k \\
X_{k}-X_{j} & \geq 0 &
\end{array}
$$

In this formulation variable $Z_{m d}$ indicates whether or not the makespan of priority class $\pi_{m}$ is realized on day $d$, and variable $Y_{m d}$ says which fraction of day $d$ passes before the makespan is realized. The parameter $\mathrm{Cmd}_{d}$ denotes the cumulative man-days offered upto day $d$, whereas $\mathrm{Amd}_{d}$ gives the man-days offered on day $d$. The total of required man-days for priority class $\pi_{m}$ is given by parameter $\mathrm{Rmd}_{m}$ and the term $-\sum_{j} a_{m j} X_{j}$ compensates for the not needed man-days for 
all jobs that have been abandoned. The latter constraints stipulate that the total abandonment cost should not exceed the available Budget, and that a task can be abandoned only if all its successors are being abandoned as well.

Computation of this lower bound has several results. First it gives a proper lower bound on the schedule cost. Second it provides a selection of tasks to be abandoned. It therefore serves as a starting point in constructing good schedules. After computing the lower bound, we only consider schedules for the tasks that have not been abandoned in the lower bound computation.

Note that the lower bounds depend on the prefixed order of priority class completions. Therefore only the lowest of the six lower bounds (corresponding with 6 permutations) forms a true lower bound on the scheduling cost.

Further note that if we have found a schedule of $\operatorname{cost} C^{*}$, and if permutation $\left(\hat{\pi}_{1}, \hat{\pi}_{2}, \hat{\pi}_{3}\right)$ yields a lower bound $\hat{L}$ such that $\hat{L} \geq C^{*}$, then we do not have to consider schedules with priority makespans in this order because cheaper solutions with this makespan order provably do not exist. In this way the lower bound helps us in not wasting our precious computation time in unprofitable directions.

It turns out that in some cases the computed lower bound is much smaller than the best solution found. This is usually caused by the fact that the real bottleneck is formed by a subset of expert technicians. It is possible to obtain stronger lower bounds than the one described above, by going through the same analysis, with the exception that the minimum number of required technicians (as provided by the first procedure) and the number of available technicians per day are restricted to those technicians that contribute positively to some specific subset of skills. It is not easy to detect which set of skills will lead to a better lower bound. This approach might obtain better bounds for instances $B 5, X 1$ and $X 5$, for instance. In instance $B 5$ the lower bound could be raised to nearly 79000 , by considering the demand for technicians that had a non-zero expertise in domain number 7 . There are only a few technicians that give a contribution and there were relatively many tasks needing expertise in this particular domain. Taking this into account requires computation of alternative $\mu^{\prime}(j), \operatorname{Amd}^{\prime}(d), \operatorname{Cmd}^{\prime}(d), \operatorname{Rmd}^{\prime}(m)$ and $a_{m j}^{\prime}$ values, restricting everything to technicians skilled in domain 7 .

\subsection{Constructing solutions}

In short, the construction algorithm is based on a strategy of building a solution from scratch, starting at day 0 , assigning a number of jobs in parallel to teams of technicians. These teams are being built on the fly. For each day, the technicians that are clustered to a team stay together. The basic ingredient of the algorithm is a model of matching a number of available jobs (those for which the predecessors have been scheduled) to a number of teams. here, teams may also refer to a group consisting of a single technician. Initially, at the beginning of each day, each team consists of one available technician. The set of jobs is either the complete set of available jobs, or the set of available jobs with a certain priority class. For the larger instances the set of candidate jobs had to be reduced to a manageable size. 
This was done by sorting the candidate jobs according to a certain SortingRule, and then splitting the sorted sequences into almost equal size parts of approximately 50 jobs. The default SortingRule was based on task-difficulty. One task can be considered more difficult than another if it requires more expertise, if it requires more technicians, if it is part of a longer chain of tasks etcetera. Different ways of quantifying these features lead to different SortingRules.

The ILP formulation has the following form. Here team $\tau$ 's skill $\underline{S}^{\tau}$ is sum of technician skills over all technicians assigned to this team; its load $\lambda(\tau)$ is current work load, that is the total duration of all tasks already assigned to the team; the team processes its assigned tasks in the order in which they have been assigned, without any idle time. Task $j$ cannot start earlier than $e(j)$. This earliest starting time (within the day) is based on the completion time of predecessors of task $j$ that have already been assigned to a team in the current day. More than one team can be assigned to a task, but we do not allow two teams that have tasks with potential successors to be combined. By this we are sure that precedence relations are not violated. For this purpose, let $\hat{T}$ denote the set of teams that process at least one task $j$ with a successor.

Maximize $\sum_{\tau} \sum_{j} C_{\tau j} X_{\tau j}+\sum_{j} G_{j} Y_{j}$ subject to:

$$
\begin{aligned}
\sum_{j} X_{\tau j} & \leq 1 & & \forall \tau \\
\sum_{\tau} \underline{S}_{i}^{\tau} X_{\tau j} & \geq \underline{R}_{i}^{j} Y_{j} & & \forall i=1, \ldots, n \\
\sum_{\tau} \lambda(\tau) X_{\tau j} & \leq(120-d(j)) Y_{j} & & \forall j \\
\sum_{\tau} \lambda(\tau) X_{\tau j} & \geq e(j) Y_{j} & & \forall j \\
X_{\tau j} & \leq Y_{j} & & \forall \tau, j \\
\sum_{\tau \in \hat{T}} X_{\tau j} & \leq Y_{j} & & \forall j \\
X_{\tau j} & \in\{0,1\} & & \forall \tau, j \\
Y_{j} & \in\{0,1\} & & \forall j .
\end{aligned}
$$

In the resulting matching problem a number of teams can be assigned to a job only if the combined expertise of the teams meets the requirements of the job. A variable $X_{\tau j}$ equals 1 if team $\tau$ is assigned to task $j$ and zero otherwise. The expertise of a team can be written as the sum of the expertises of the technicians that are in the team. A team is assigned to at most one job (in each matching iteration) and a job may or may not be selected. The variable $Y_{j}$ is 1 only if task $j$ is selected. It can only be selected, if it is simultaneously assigned a set of teams the union of which is competent with respect to the requirements of task $j$. The additional restrictions enforce that a selected task is processed immediately after a team has finished its already assigned work, without the introduction of idle time, and without breaking any precedence constraint. The assignment and selection problem bears much resemblance with capacitated facility location problems.

The objective is to select a 'nicest' combination of jobs. For measuring the quality of an assignment we consider several MatchingObjectives defined by setting coefficients $C_{\tau j}$ and $G_{j}$ to appropriate values. These values are based on job length, job load, job difficulty or job priority or a combination of these. A default setting 
is choosing $C_{\tau j}=0$, and $G_{j}=d(j) * \mu(j)$. This reflects the desire to schedule as much work as early as possible. The problem is formulated as a linear integer programming problem and passed to the solver (CPLEX).

A further characteristic that influences the outcome is found in the way the ILP-model of the matching problem is formulated and passed to the solver. One reason is that the solver is given only a limited amount of time. It may therefore have to break off its branch-and-bound search prematurely. A second reason is that there may be more than one optimal solution. In case the coefficients $C_{\tau j}=C_{\tau}$ are task-independent, the model has a sparse formulation which tends to solve more quickly, and a dense formulation which seems to have a tendency of producing slightly better solutions (in terms of complete day schedules and use of scarce resources). By introduction of a slack variable $\sigma_{\tau}$, the first inequality of the model can be converted into an equation: $\sum_{j} X_{\tau j}+\sigma_{\tau}=1 \forall \tau$, and then the variables $X_{\tau j}$ may be (partially) deleted from the objective function. In the algorithm we may use different values for the SparsityFactor (between 0.0 and 1.0). This refers to the fraction of inequalities for which the above conversion is implemented.

Obviously, for the instances in which part of the jobs can be abandoned, it makes quite a difference for the resulting schedule value, which set of jobs is abandoned. We consider different objectives for selecting jobs to be abandoned. Restrictions are that the cost of abandoning jobs must be within the budget, and secondly that if a job is being abandoned, then also its successors should be abandoned. The selection of abandoned jobs further influences the lower bound on the schedule cost (for the remaining jobs). Therefore we consider several LowerBoundObjectives. They reflect the bonus obtained by skipping certain jobs. Again this depends on length, load, priority, difficulty of jobs, or of combinations of these.

Finally, one can try to obtain a solution as close as possible to the computed lower bound. To this purpose it may be necessary to temporarily 'upgrade' some jobs: if $J_{1} \rightarrow \ldots \rightarrow J_{k}$ is a chain of jobs all of priority 4 , and the total length is higher than the difference of the relaxed makespans $C_{\max }^{4}-C_{\max }^{1}$, we should complete job $J_{1}$ earlier than $C_{\max }^{1}$. We may therefore change its priority class to 1 , before we call the schedule construction algorithm. We consider two levels of UpdatingPriorities (yes/no).

\subsection{Creating multiple solutions}

In principle there are two ways to generate many solutions greedily according to the above scheme. One is to introduce randomness in the ordering and randomness in the matching objective coefficients. We have chosen not to do this but only vary the SortingRules, the LowerBoundObjectives, the MatchingObjectives, the UpdatingPriorities over a limited set of parameter settings. By doing this we limit ourselves to the creation of at most $6 \cdot 4 \cdot 3 \cdot 3 \cdot 2=432$ schedules. The factor 6 of course results from the different permutations of the priority class makespan orderings. Also one could consider adding a post-processing stage to polish the 
obtained schedules. Most of the solutions found in the earliest stages already show good quality.

\section{Reflection And PROspects}

The use of linear programming techniques in the area of schedule construction poses the potential user for several decision questions where effectivity and computation time have to traded off. In particular the fact that the algorithm is allowed a limited amount of time can cause difficulties.

\subsection{TIME MANAGEMENT}

In the area of local search which is a more standard approach to complex scheduling problems, one usually defines a construction heuristic to come up with a first schedule, and next a search strategy is employed to search the solution space as long as time permits. A variant is one in which the construction heuristic is adapted to the observed solutions, and is repeated again and again.

For integer linear programming models it is very much harder to predict the duration of certain calculations. Branch-and-bound solution methods may end fairly quickly, but may also get stuck in a deep search tree and never end.

For the problems at hand we found the first two problems preprocessing and lower bounds to behave rather well and we could solve those to optimality without having to set time limits. The team-job assignment problem however was much harder to handle. The problems in set $A$ could be handled in their complete formulation - as far as size was concerned. The running times were rather unpredictable. Problems in set $B$ were already so big that finding a first solution could already take much time, and waiting for an optimal answer was out of the question. This problem was resolved by ordering tasks from highly to less important, selecting subsets of 50-80 consecutive tasks, and trying to solve the matching problem with this limited set of candidate tasks. Furthermore it was necessary to set rather small time limits $(5-10 \mathrm{~s})$ on the MIP solver, for each matching problem.

For the future we may want to design algorithms capable of rescheduling or of on-line scheduling, based on this MIP approach.

\subsection{LOWER BOUND QUALITY}

As mentioned before, in some cases the lower bound deviates a lot from the best solution found. In some cases this is caused by a critical set of scarce expertise skills, or by experts only available a part of the time. There is some room for improvement.

\subsection{Flat objective}

The objective by which schedule quality is measured is rather flat. There is not yet a good understanding of how to prioritize tasks so as to have low priority class 
makespans. Of course this difficulty holds also for standard local search methods. It would also be interesting to see how this LP approach will perform when other quality measures are being used, for instance weighted sum of completion times, over all jobs.

\subsection{Constraints}

The complex restriction of having enough qualified technicians in a team that handles a task is very well suited for our Mixed Integer Linear program approach. We may further study more complex restrictions, such as providing for stable assignments. We will further collaborate with France Telecom to investigate many other features of maintenance schedules. For now the conclusion is that MIP methods seem to promise a lot of possibilities.

\section{REFERENCES}

[1] D. Applegate and W. Cook, A computational study of the job-shop scheduling problem. ORSA J. Comput. 3 (1991) 149-156.

[2] E.L. Lawler and J. Labetoulle, On preemptive scheduling of unrelated parallel processors by linear programming. J. Assoc. Comput. Mach. 25 (1978) 612-619.

[3] Y. Pochet and Laurence A. Wolsey, Production planning by mixed integer programming. Springer Series in Operations Research and Financial Engineering. Springer, New York (2006) xxiv +499 p. 